HEAD AND NECK

\title{
Mandibular reconstruction using a new design for a patient-specific plate to support a fibular free flap and avoid double-barrel technique
}

\author{
Ricostruzione mandibolare con lembo di fibula supportato da nuova tipologia \\ di placca personalizzata al fine di evitare la tecnica della doppia barra
}

\author{
Achille Tarsitano ${ }^{1}$, Salvatore Battaglia ${ }^{1}$, Giuseppe Corinaldesi² ${ }^{2}$ Claudio Marchetti' ${ }^{1}$ Gerardo Pellegrino², Leonardo Ciocca $^{2}$ \\ ${ }^{1}$ Maxillofacial Surgery Unit, Head and Neck Department, IRCCS Azienda Ospedaliera Universitaria di Bologna, Department of \\ Biomedical and Neuromotor Sciences, Alma Mater Studiorum University of Bologna; 2 Section of Oral Surgery, Department of \\ Biomedical and Neuromotor Science, Alma Mater Studiorum University of Bologna
}

\begin{abstract}
SUMMARY
Mandibular reconstruction is a primary concern for head and neck reconstructive surgeons because of the aesthetic restoration needs after ablative surgery, as well as for functional reasons: the mandible has a central functional role in speaking, swallowing and mastication. It is generally agreed that the gold standard for mandibular reconstruction is a bone free flap supported by a reconstructive titanium plate. The fibular flap represents the first choice for multi-segment mandibular reconstruction. The fibula, harvested as a single barrel graft, does not exhibit sufficient thickness to reach the original height of the native mandible; therefore, the positioning of dental implants is often deeper than that of the native alveolar crest. The aim of this study was to evaluate the positioning of the fibular free flap as it pertains to the restoration of vertical mandible height, by modifying the design of a 3D-printed titanium patient-specific implant (PSI). In this novel reconstructive workflow, the customised plate was projected to support the fibular flap at an alveolar bone position above the typical inferior mandibular border, and carried out on four patients. All patients were treated for benign neoplasms involving mandibular bone. Clinical outcomes and accuracy of the procedure are described. Our reconstructive proposal appears to be a valid alternative to the double-barrel technique in order to restore the vertical height of the reconstructed mandible.
\end{abstract}

KEY WORDS: mandibular reconstruction, customised plate, fibula free flap, double-barrel technique, head and neck surgery

\section{RIASSUNTO}

La ricostruzione mandibolare è particolarmente rilevante per il chirurgo cervico-cefalico, in quanto influenza significativamente $i$ successi in termini di risultati estetici e funzionali per il paziente. Il gold standard per tale ricostruzione è l'utilizzo di lembi ossei rivascolarizzati, stabilizzati mediante una placca ricostruttiva in titanio. Il lembo di fibula rappresenta la prima scelta ricostruttiva laddove necessitino molteplici segmenti ossei. Tuttavia questo lembo, allestito secondo la tecnica della singola barra, non permette di ripristinare l'altezza mandibolare nativa, adeguata per una corretta riabilitazione masticatoria implanto-supportata. Lo scopo di questo studio è quindi presentare un nuovo design di placca ricostruttiva custom-made, atta a posizionare il segmento osseo di fibula in una posizione più coronale, evitando pertanto la necessità di allestire il lembo secondo la tecnica della doppia barra. La placca custom-made contribuisce inoltre al mantenimento del profilo mandibolare, garantendo il ripristino morfologico della ricostruzione. Questo protocollo è stato eseguito su quattro pazienti sottoposti a resezione mandibolare per tumori benigni. I risultati clinici e di accuratezza della procedura sono presentati. Il protocollo presentato sembra una valida alternativa alla tecnica della doppia barra al fine di ripristinare l'altezza mandibolare nativa.

PAROLE CHIAVE: ricostruzione mandibolare, placca personalizzata, lembo libero di fibula, tecnica della doppia barra, chirurgia della testa e del collo
Received: November 13, 2020

Accepted: April 29, 2021

Correspondence

Achille Tarsitano

S. Orsola University Hospital, Head and Neck Department, DIBINEM - University of Bologna

via Massarenti 9, 40100 Bologna, Italy

Tel. +390512142463

E-mail: achille.tarsitano2@unibo.it

Funding

None.

Conflict of interest

The Authors declare no conflict of interest.

How to cite this article: Tarsitano A, Battaglia S, Corinaldesi G, et al. Mandibular reconstruction using a new design for a patientspecific plate to support a fibular free flap and avoid double-barrel technique. Acta Otorhinolaryngol Ital 2021;41:230-235. https://doi. org/10.14639/0392-100X-N0549

๑ Società Italiana di Otorinolaringoiatria e Chirurgia Cervico-Facciale

\section{(c) (1) $(2)$}

This is an open access article distributed in accordance with the CC-BY-NC-ND (Creative Commons Attribution-NonCommercial-NoDerivatives 4.0 International) license. The article can be used by giving appropriate credit and mentioning the license, but only for non-commercial purposes and only in the original version. For further information: https:// creativecommons.org/licenses/by-nc-nd/4.0/deed.en 


\section{Introduction}

Mandibular reconstruction is a primary concern for head and neck reconstructive surgeons because of the aesthetic restoration needs after ablative surgery, as well as for functional reasons: the mandible has a central functional role in speaking, swallowing and mastication. It is generally agreed that the gold standard for mandibular reconstruction is a bone free flap supported by a reconstructive titanium plate ${ }^{1}$. The fibular flap represents the first choice for multisegment mandibular reconstruction ${ }^{2}$.

Multiple papers ${ }^{3}$ have reported optimal anatomic and aesthetic outcomes of mandibular reconstruction after cancer ablative surgery when computer-aided design/computeraided manufacturing (CAD/CAM) technology was used; however, the prosthetic workflow to restore masticatory efficiency remains a challenge ${ }^{4}$. Although digital planning is known to be accurate, detailed and efficient, the reconstructive plate that supports the fibular segments runs along the inferior border of the mandible, thus requiring the surgeon to perform bony reconstruction at the basal bone position. The fibula, harvested as a single barrel graft, does not exhibit sufficient thickness to reach the original height of the native mandible; therefore, the positioning of dental implants is often deeper than that of the native alveolar crest, resulting in poor aesthetics and inappropriate hygiene ${ }^{1}$.

The aim of this study was to evaluate the positioning of the fibular free flap as it pertains to the restoration of vertical mandible height by modifying the design of a 3D-printed titanium patient-specific implant (PSI).

\section{Description of the clinical techniques and technology}

This study was approved by the S. Orsola Hospital Ethics Committee (approval no. 57/2011/O/Disp).

\section{D segmentation of the fibular flap}

Digital Imaging and Communications in Medicine (DICOM) format files obtained from pre-operative head and neck computed tomography (CT) and donor-site CT angiography scans of the legs were processed by the surgeons using MIMICS software (Materialise, Leuven, Belgium) in order to obtain the 3D virtual models. Resection virtual planning was performed considering radical resection of the tumour within safe margins (Fig. 1).

Simulation of reconstruction began with virtual restoration of the native mandibular profile and contour. The reconstructive procedure was virtually performed on 3D objects obtained from DICOM files of the donor-site CT angiography scan. During this step, the surgeon chose the left or

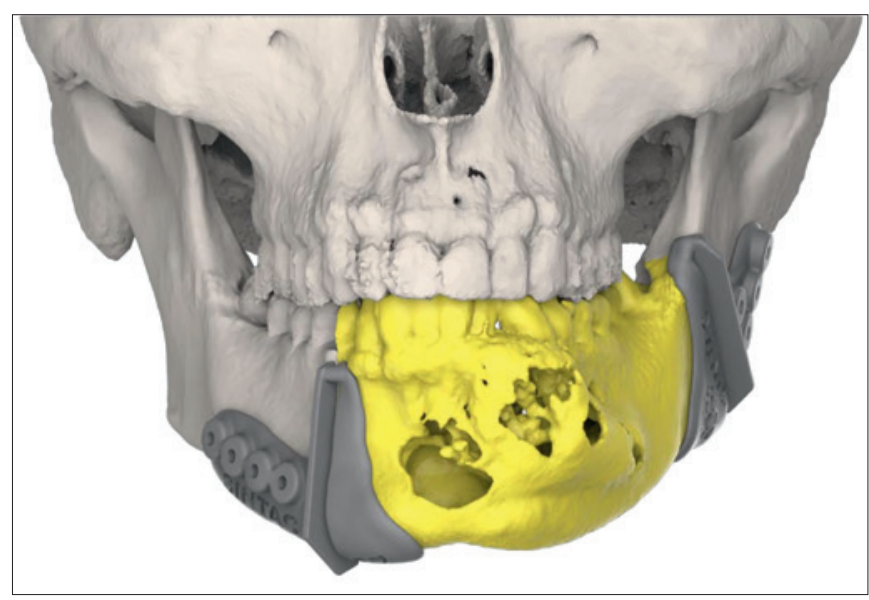

Figure 1. Tumour resection planning.

the right leg, based both on the final position of the fibula in the mandible with respect to the best bicorticalism for implants and the best anchoring surface to screw into the bone plate and on the vascular pedicle anatomy (Fig. 2). Selection was also made with consideration of the number of necessary segments and the angulation of the segments within the mandibular defect, based on the appropriate pedicle length for microvascular anastomoses. The resection cutting guides and osteotomy guides for the bony free flap were designed based on resection and reconstruction planning with TRI MATIC software (Materialise, Leuven, Belgium).

\section{Occlusion-driven design of double-level anatomy}

During the simulation of reconstructive surgery, the position of teeth in the native mandibular arch is considered to preserve occlusion and function. When a unilateral edentulous area was observed in the affected side, the positions of teeth on the healthy side were mirrored and correct occlusal relationships were simulated using MIMICS software (Materialise, Leuven, Belgium). By contrast, if the contralateral arch was edentulous, a radiographic guide was constructed with radiopaque teeth and used during CT examination to allow reproduction of the teeth in their maxillomandibular relationship.

Bicorticalism is necessary for the best osteointegration and primary stability of oral implants in the fibular flap, and thus the position of the fibula was determined with respect to the occlusal plane. The three-dimensional fibula positioning was simulated in order to obtain better dental implantlbone surface contact (Fig. 2).

In our novel protocol, the design of the bone plate was modified to allow mimicry of double-level anatomy - i.e., increasingly lateral basal bone inferior mandible margin 


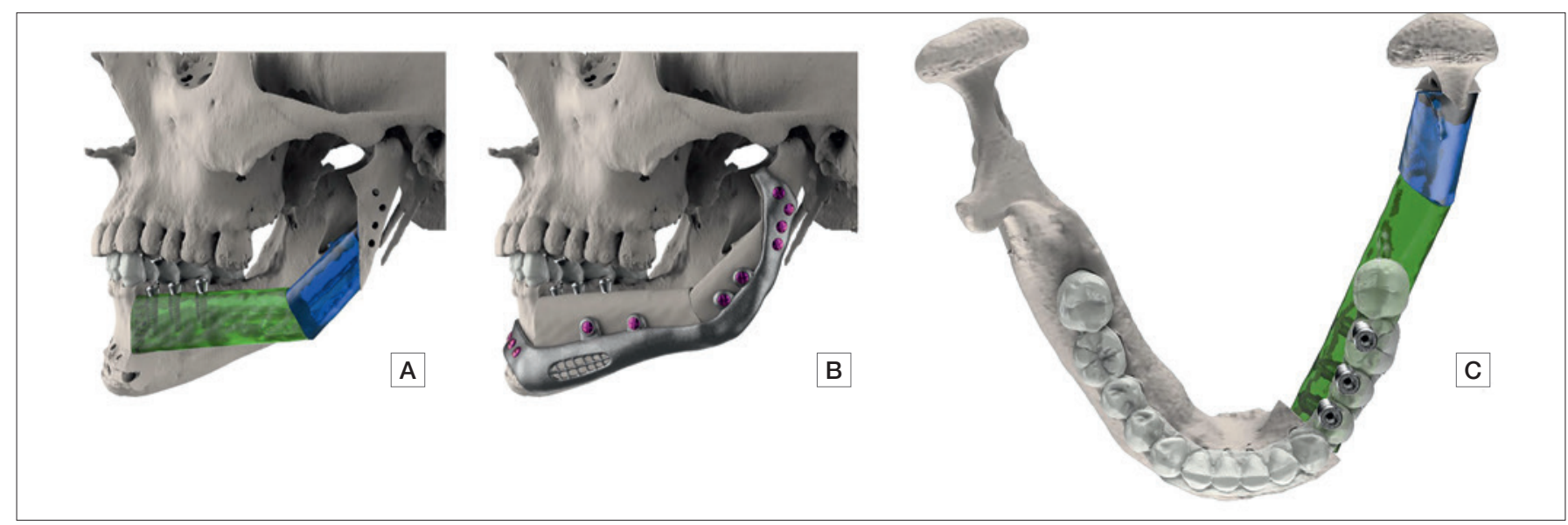

Figure 2. Positioning of the fibula as related to occlusion, implants and alveolar bone height from lateral $(A, B)$ and occlusal views (C).

and increasingly medial alveolar bone position. Therefore, the fibula reproduced alveolar bone. Otherwise, the inferior border was reproduced by the plate. Two or more vertical arms were designed from the top border of the bone plate to support the bony segment and secure it in the necessary upper position.

\section{Design of the customised reconstructive plate}

In this novel CAD/CAM workflow, the customised plate was projected to support the fibular flap at an alveolar bone position above the typical inferior mandibular border. A new containing deck was designed using Geomagic Freeform Plus software (3D Systems, Rock Hill, SC, USA) to support the bony flap in the higher position; this border allowed distribution of occlusal loading onto bone screws and directly onto the PSI structure (Fig. 3). Therefore, the customised plate fully restored the mandibular bony shape of the inferior border, while allowing the surgeon to position fibular segments in the alveolar region, providing a more feasible and aesthetic contour (Fig. 4).

On the inferior portion of the PSI, a grid was designed to allow the repositioning of oral floor muscles at the original bone insertion (Fig. 4). The plate had a $2.4 \mathrm{~mm}$ thickness and was fixed to the native mandible using a $2.4 \mathrm{~mm}$ fixation system.

\section{$3 D$-printing of surgical devices}

At the end of the process, the surgeons validated and verified the project with MAGICS software (Materialise, Leuven, Belgium). The Standard Tessellation Language (STL) interface format files of the customised reconstructive plate were processed, and the plate was then manufactured via direct metal laser sintering with a DMLS M280 system (Electro-Optical Systems, GmbH, Krailling, Germany) us-

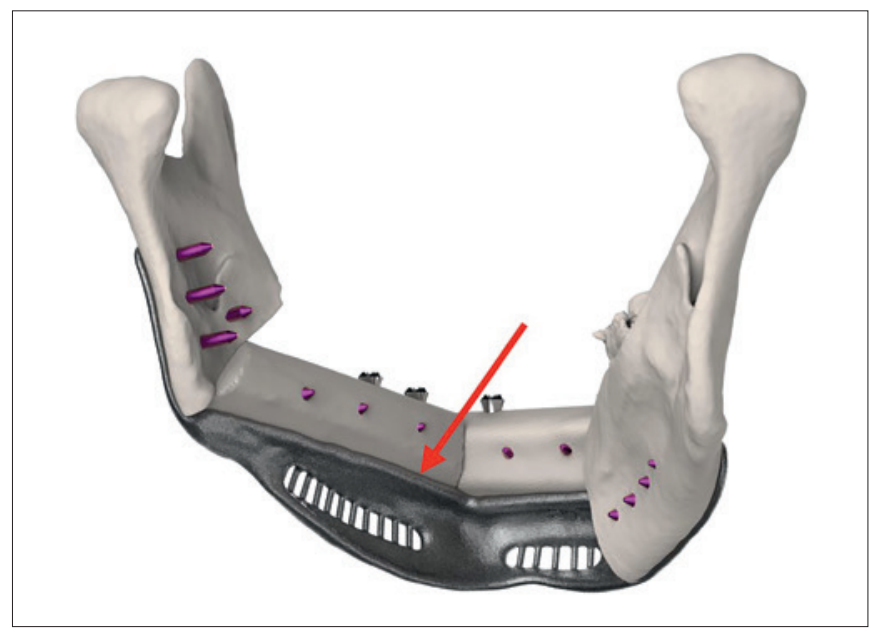

Figure 3. Virtual design of the containing deck for sustaining the fibular bone segments (red arrow).

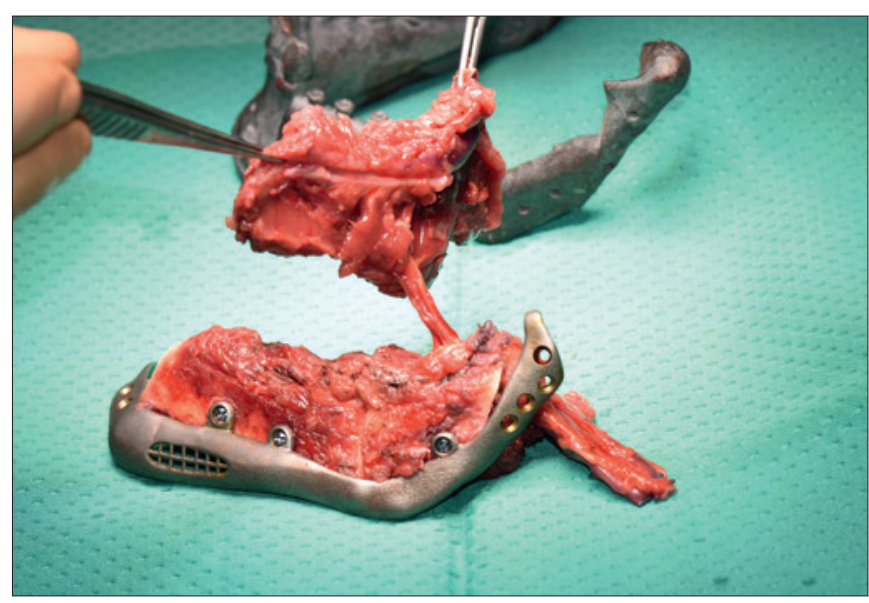

Figure 4. Mandibular reconstructive plate design according to the native mandibular anatomy. 
ing a titanium alloy with an additive-in layer technique. In the same manner, laser sintering following the STL files of the resection and fibular cutting guides was performed using the SLS FORMIGA P110 system (Electro-Optical Systems, GmbH, Krailling, Germany).

\section{Fibula free flap harvesting}

For all cases, the fibula has been harvested both as exclusively bone flap and as composite osteo-muscular flap. Since all recruited patients were affected by benign bone tumours, no large amount of soft tissues removal has been required and no need for skin paddle harvesting was identified. Oral mucosal lining was preserved for all patients in order to improve dental rehabilitation in the future implants. A fibular cutting guide was used to segment the bony flap. The guide was fixed to the fibula using 2.0 mono-cortical screws. It allowed accurate bone segmentation, as pre-planned. Also, the holes in the fibular cutting guides were in the same locations as those of the reconstructive plates, allowing the surgeon to correctly and rapidly place bony segments during reconstruction.

\section{Clinical outcomes}

The previously described protocol was carried out on four patients. All patients were treated for benign neoplasms in- volving mandibular bone. The mandibular symphysis was resected in two of the patients. Mandibular resection was completed in accordance with resection virtual planning, using cutting guides. Fibular segmentation and flap insertion were performed in accordance with virtual planning in all patients. The average follow-up was 12 months. No intraoperative complications were recorded. No post-operative complications were registered in terms of inflammation or plate exposure.

\section{Evaluation of fibular positioning accuracy}

To verify fibular positioning and angulation, the STL file used for virtual planning and the STL file of the postoperative CT scan were imported into a mesh-processing software using MeshLab (Visual Computing Lab - ISTI-CNR, Pisa, Italy). After alignment of the two resulting meshes, the software automatically overlapped the meshes based on fixed reference landmarks (screw holes on the plate). Reconstruction accuracy was determined by evaluating the average error of fibular unit inclination, relative to the horizontal plane (craniocaudal inclination) (Fig. 5).

The mean error of total reconstruction among all patients was $1.2 \mathrm{~mm}$ (range $0.8-1.3 \mathrm{~mm}$ ). The mean surface error of PSI intraoperative positioning was $0.86 \mathrm{~mm}$ (range, $0-2.1 \mathrm{~mm}$ ).
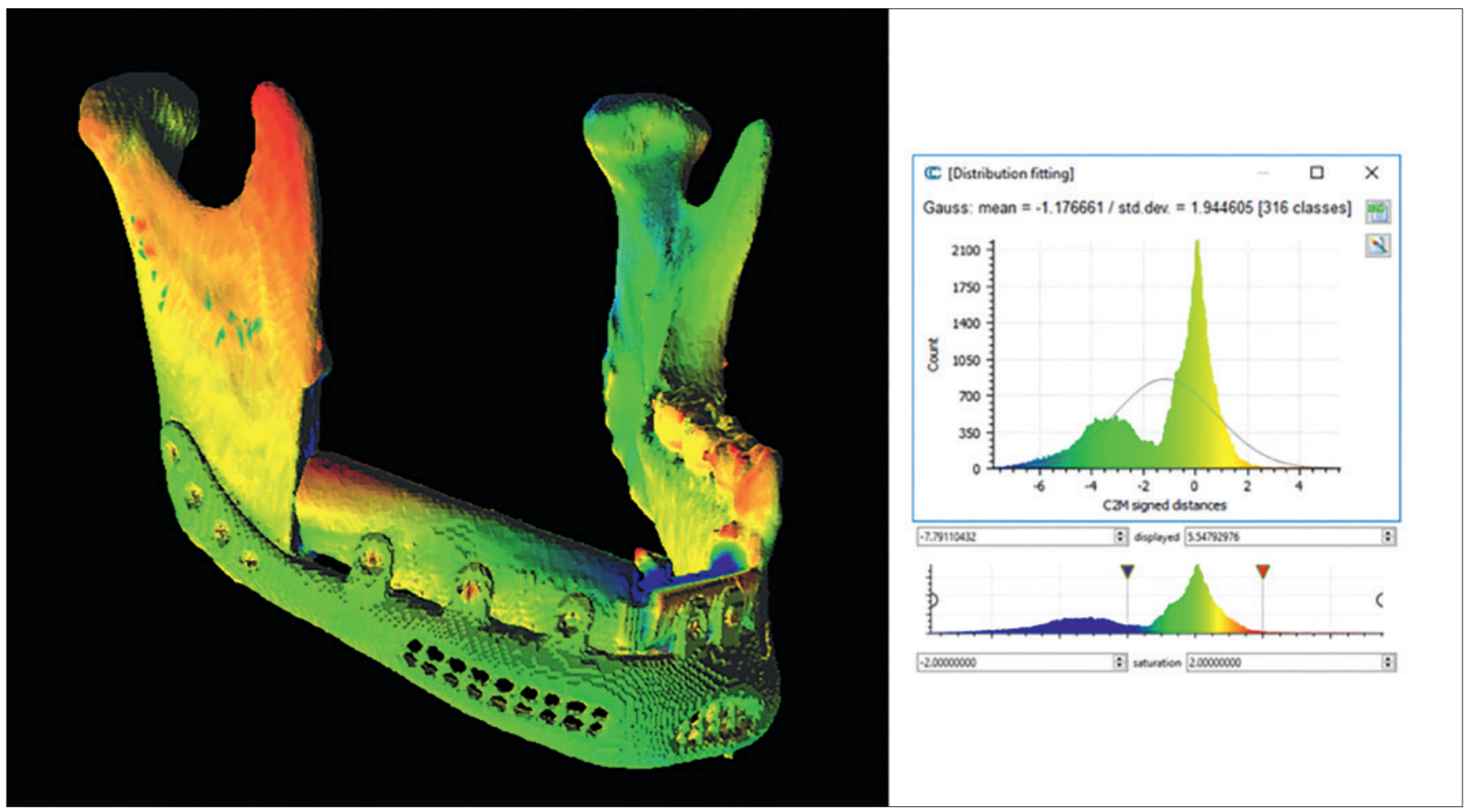

Figure 5. Accuracy assessed by colour map obtained from superimposition between pre-operative planning and post-operative CT-scan. 


\section{Discussion}

Repair of long-span mandibular defects with a free fibular flap is now a routine procedure. Several studies have provided evidence regarding the accuracy of CAD/CAM technology applied to mandibular reconstruction with fibular free flap harvested as single graft ${ }^{4}$. In fact, a fibular flap is typically projected to restore the basal bone to preserve the mandibular contour and maintain correct anatomy of the facial profile. However, the bone height of the neomandible after reconstruction with a fibular flap is about half that of the dentulous mandible. When a fibular graft is placed only at the inferior border of the mandible, the resulting vertical discrepancy between the graft segment and the occlusal plane can adversely affect implant mechanics or denture stability and retention.

To overcome this problem, many surgical solutions have been proposed:

1. A double-barrel fibular flap can be harvested.

2. A different type of bony free flap as well as scapula or iliac crest flap can be used.

The double-barrel fibular flap presents the advantage of best restoring the correct alveolar and basal height, but sometimes the surgical risks of reduced blood supply due to bending may lead to vascular complications. Another limit still existing for both double barrel fibular flap and other bony free flaps is the length of mandibular arch to restore. For mandibular defects larger than $10 \mathrm{~cm}$, many authors do not suggest a double-barrel technique because the fibula length usually required is excessive, which would result in shortening too much the pedicle ${ }^{1}$. However, both for lateral and moderate mandibular defects it remains the first reconstruction option. Virtual surgical planning can allow surgeons to better assess bone segments positioning ${ }^{4}$. Alternatives to the double-barrel fibular flap may be folded scapula or iliac crest free flaps. The first appears to be valid donor sites for cases with large soft tissue and minimal bone requirements ${ }^{1}$. Differently, the iliac crest free flap is limited by short vascular pedicle, higher morbidity at donor site and restricted bone length ${ }^{1}$. The fibula donor site remains the first choice for cases with large bony defects requiring multiple osteotomies and for dental rehabilitation ${ }^{1}$. In this report, we propose a modification of the design of the mandibular reconstructive plate in order to position the fibular bone segments at the alveolar level, avoiding the double-barrel technique harvesting.

The principal advantage of this technique is the fibular positioning to avoid the vertical discrepancy between the graft segment and the native mandible. The inferior border of the facial profile can be maintained as the bone plate is projected to reproduce the inferior border of the mandible using metal, simulating native anatomy whenever possible, or mirroring the healthy side when a unilateral disease-affected site was present. Furthermore, the alveolar portion of mandibular bone can be restored.

Disadvantages of this technique include the cost of CAD/ CAM technology and confinement of this technique to the treatment of benign tumours.

Regarding this first issue, many approaches to minimising costs while simultaneously optimising patient treatment are potentially available. The principal area for such efforts is the theatre, as reducing the operating time can considerably affect the overall cost ${ }^{5}$. In a previously published paper, we estimated that our institutional cost per minute of theatre time was $€ 30$. Considering that the average time gained using CAD-CAM surgery was $115.5 \mathrm{~min}$, the money saved resulting from the time gain was $€ 3,450$. This cost corresponds approximately to the total price of the CAD-CAM surgery ${ }^{5}$. Another area that should be considered is the rate of complications experienced by mandibular reconstruction patients who undergo the CAD-CAM technique versus the freehand technique. In fact, complications can add costs in terms of needing secondary revision procedures or hospitalisation. Thus, CAD-CAM surgery can be considered as a useful tool to improve the quality of the reconstruction and reduce the need for secondary revision procedures (flap repositioning for occlusion restoration or plate dislocation). Moreover, guided surgery has the potential not only to reduce surgical time, but also to reduce ischaemic time for the fibular flap ${ }^{5,6}$.

Regarding the second issue, we have to highlight that the amount of titanium needed for this type of restoration can increase the risk of plate exposure after radiotherapy.

For this reason, we propose this technique for patients affected by benign tumours. Further studies are needed to determine whether this protocol is useful in treating patients with malignant cancer, as well as to determine the longterm outcomes of this approach.

\section{Acknowledgements}

The authors thank Andrea Sandi for his valuable work on CAD.

\section{References}

1 Anne-Gaëlle B, Samuel S, Julie B, et al. Dental implant placement after mandibular reconstruction by microvascular free fibula flap: current knowledge and remaining questions. Oral Oncol 2011;47:10991104. https://doi.org/10.1016/j.oraloncology.2011.07.016

2 Ferri A, Varazzani A, Bolzoni AR, et al. A multicenter survey on_computer-aided design and computer-aided manufacturing mandibular reconstruction from Italian community. Microsurgery 2019;39:673674. https://doi.org/10.1002/micr.30505 
3 Tarsitano A, Ciocca L, Cipriani R, et al. Mandibular reconstruction using fibula free flap harvested using a customised cutting guide: how we do it. Acta Otorhinolaryngol Ital 2015;35:198-201.

4 Tarsitano A, Ciocca L, Scotti R, et al. Morphological results of customized microvascular mandibular reconstruction: a comparative study. J Craniomaxillofac Surg 2016;44:697-702. https://doi. org/10.1016/j.jcms.2016.03.007
5 Tarsitano A, Battaglia S, Crimi S, et al. Is a computer-assisted design and computer-assisted manufacturing method for mandibular reconstruction economically viable? J Craniomaxillofac Surg 2016;44:795799. https://doi.org/10.1016/j.jcms.2016.04.003

6 Sweed AH, Bolzoni AR, Kadubiec A, et al. Factors influencing $\mathrm{CAD} / \mathrm{CAM}$ accuracy in fibula free flap mandibular reconstruction. Acta Otorhinolaryngol Ital 2020;40:138-143. https://doi. org/10.14639/0392-100X-N0400 